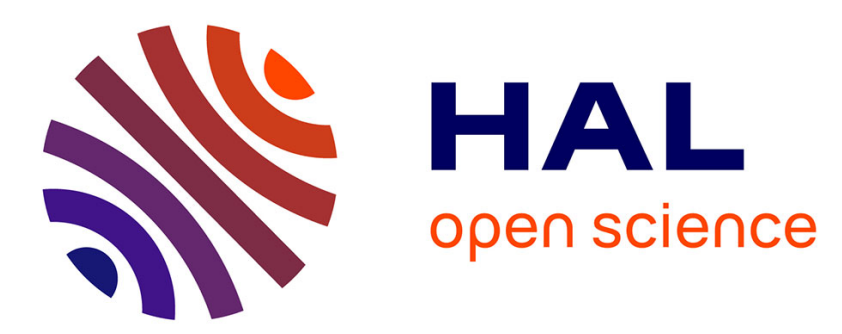

\title{
Activation of surface oxygen sites on an iridium-based model catalyst for the oxygen evolution reaction
}

Alexis Grimaud, Arnaud Demortiere, Matthieu Saubanere, Walid Dachraoui, Martial Duchamp, Marie-Liesse Doublet, Jean-Marie Tarascon

\section{- To cite this version:}

Alexis Grimaud, Arnaud Demortiere, Matthieu Saubanere, Walid Dachraoui, Martial Duchamp, et al. Activation of surface oxygen sites on an iridium-based model catalyst for the oxygen evolution reaction. Nature Energy, 2017, 2 (1), pp.1-10. 10.1038/nenergy.2016.189 . hal-03315781

\author{
HAL Id: hal-03315781 \\ https://hal.science/hal-03315781
}

Submitted on 5 Aug 2021

HAL is a multi-disciplinary open access archive for the deposit and dissemination of scientific research documents, whether they are published or not. The documents may come from teaching and research institutions in France or abroad, or from public or private research centers.
L'archive ouverte pluridisciplinaire HAL, est destinée au dépôt et à la diffusion de documents scientifiques de niveau recherche, publiés ou non, émanant des établissements d'enseignement et de recherche français ou étrangers, des laboratoires publics ou privés. 


\title{
Activation of surface oxygen sites on an iridium-based model catalyst for the oxygen evolution reaction
}

\author{
Alexis GRIMAUD, ${ }^{1,2^{*}}$ Arnaud DEMORTIERE, ${ }^{2,3}$ Matthieu SAUBANERE, ${ }^{1,2,4}$ Walid DACHRAOUI, ${ }^{2,3}$ Martial \\ DUCHAMP, ${ }^{5}$ Marie-Liesse DOUBLET $T^{2,4}$ and Jean-Marie TARASCON ${ }^{1,2,6,7}$
}

1. Chimie du Solide et de l'Energie, UMR 8260, Collège de France, 75231 Paris Cedex 05, France

2. Réseau sur le Stockage Electrochimique de l'Energie (RS2E), FR CNRS 3459, 80039 Amiens Cedex, France

3. Laboratoire de Réactivité et Chimie des Solides, UMR CNRS 7314, 33 Rue Saint Leu, 80039 Amiens Cedex, France

4. Institut Charles Gerhardt, CNRS UMR 5253, Université Montpellier, Place E. Bataillon, 34095 Montpellier, France

5. Ernst Ruska-Centre for Microscopy and Spectroscopy with Electrons (ER-C) and Peter Grünberg Institute (PGI), Forschungszentrum Jülich, 52428 Jülich, Germany

6. ALISTORE-European Research Institute, FR CNRS 3104, 80039 Amiens, France

7. Sorbonne Universités - UPMC Univ Paris 06, 75005 Paris, France

Corresponding author: alexis.grimaud@college-de-france.fr

\section{Abstract:}

The oxygen evolution reaction (OER) is of prime importance in multiple energy storage devices, however, deeper mechanistic understanding is required in order to design enhanced electrocatalysts for the reaction. Current understanding of the OER mechanism based on oxygen adsorption on a metallic surface site fails to fully explain the activity of iridium and ruthenium oxide surfaces, and the drastic surface reconstruction observed for the most active OER catalysts. Here we demonstrate, using $\mathrm{La}_{2} \mathrm{LilrO}_{6}$ as a model catalyst, that the exceptionally high activity found for Ir-based catalysts arises from the formation of active surface oxygen atoms that act as electrophilic centres for water to react. Moreover, we observe with the help of transmission electron microscopy drastic surface reconstruction and iridium migration from the bulk to the surface. Therefore, we establish a correlation between surface activity and surface stability for OER catalysts which is rooted in the formation of surface reactive oxygen. 


\section{Introduction}

The design of new, active and cost-effective heterogeneous catalysts for the oxygen evolution reaction (OER) has been the subject of intense researches for decades. ${ }^{1,2}$ One group of OER catalysts with promising performance are transition metal oxides (TMOs) with the perovskite structure. ${ }^{3-9}$ Despite much research, the discovery of new active compounds is rather slow, which can be understood by the lack of pertinent descriptors able to capture the physical origin of the OER activity that chemists could use as a guideline to design new catalysts. Hence, much effort has been made to rationalise OER activity. ${ }^{8-11} \mathrm{~A}$ common approach was so far to assume that the surface is stable and provides transition metal adsorption sites for oxygen evolution to proceed, with the best surface binding oxygen neither too strongly nor too weakly, following Sabatier's principle. ${ }^{10,12}$ For instance, the OER activity of perovskites in alkaline conditions was shown to correlate with the occupation of the $\mathrm{e}_{\mathrm{g}}$-like $\sigma^{*}$ states, ${ }^{8,13}$ suggesting that a direct $\sigma$ interaction with a metallic state is necessary for oxygen to adsorb. ${ }^{14}$ This suggestion has recently been challenged by recent demonstration that reconstruction and/or amorphization processes on the surface of the most active TMOs during the OER can occur, ${ }^{15-17}$ as well as cationic dissolution for Ir- and Ru-based oxides. ${ }^{18-21}$ Nevertheless, it remains that neither the very large OER activity found for oxidized surfaces such as hydrated $\mathrm{IrO}_{2}$ or $\mathrm{RuO}_{2}$ for which the $\mathrm{e}_{\mathrm{g}}$-like $\sigma^{*}$ states are empty, nor the exact nature of the active site, has yet being explained. ${ }^{22-24}$

Despite this complexity, it seems that a consensus is met between theoretical calculations and experimental works, ${ }^{6,8-10,12}$ with the TMOs based on late transition metals with high oxidation states being among the most active catalysts. While still incomplete, the current understanding is the following. ${ }^{9,25}$ By decreasing the chemical potential of the transition metal, the TM-O bonds become more covalent and therefore weaker in energy, ${ }^{26}$ hence lowering the energy associated with O-O bond formation $\left({ }^{*} \mathrm{O}+\mathrm{H}_{2} \mathrm{O} \rightarrow{ }^{*} \mathrm{OOH}+\mathrm{H}^{+}+\mathrm{e}^{-}\right)^{9}$ usually found to be the rate determining step for the OER. ${ }^{12}$ Nevertheless, other mechanisms can arise when triggering the formation of reactive oxygen 
atoms on the surface of TMOs, including their involvement into the formation of molecular oxygen, ${ }^{27-29}$ situation normally encountered for homogeneous catalysts for which activation and bond-breaking/formation processes are the underlying steps. ${ }^{14,30}$ Therefore, it is not surprising that surface degradation can occur for highly active TMO catalysts. ${ }^{15-17,31}$ Although this observation represents a step forward in the development of OER catalysts, the exact interplay existing between the surface oxygen activation, the OER activity and the surface reconstruction is not well understood yet.

In contrast to homogeneous molecular catalysts for which the active site can be carefully designed and explored, the study of the active site in TMO heterogeneous catalysts is rather complex due to the lack of analytical tools to probe such transient states. In this work, we alleviate this complexity by designing a TMO catalyst with a well-defined redox potential and show how the modification of the adsorption site from a metallic to an oxygen site following an oxidation process affects the OER activity and the catalyst stability. While the $\mathrm{A}^{3+} \mathrm{M}^{3+} \mathrm{O}_{3}$ (A being a lanthanide) and $\mathrm{A}^{2+} \mathrm{M}^{4+} \mathrm{O}_{3}$ (A being an alkaline earth) perovskites have been widely studied as OER catalysts, higher oxidation states can be stabilised by the substitution of monovalent alkaline metal on the transition metal site to form $\mathrm{La}_{2} \mathrm{LiMO}_{6}$ with $\mathrm{M}$ being Ir and $\mathrm{Ru}^{32,33}$ In this study, we demonstrate that metallic $\mathrm{t}_{2 \mathrm{~g}}$-like $\pi^{*}$ states are inactive for OER and that the surface active site for oxidized Ir-based catalysts is a purely oxygen state which shows enhanced activity. The enhanced reactivity of surface oxygen species is at the expense of surface stability and we show that the activation of the oxygen surface active site eventually leads to iridium migration from the bulk to the surface of the catalyst, explaining the relatively poor stability observed for Ir-based catalysts.

\section{OER activity in alkaline and acidic conditions}

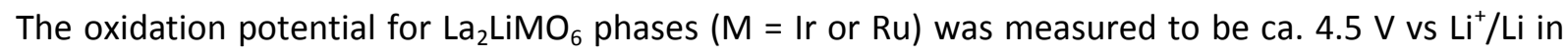
organic solvent (Supplementary Figure 1). As seen by XRD and confirmed by DFT calculations, the 
bulk structure is stable upon delithiation (Supplementary Figures 1 and 2 and Supplementary Table 2). The OER activity for both phases was measured from high $\mathrm{pH}$, to neutral $\mathrm{pH}$ and to low $\mathrm{pH}$, spanning a range of potential from 0.3 to $1.7 \mathrm{~V}$ vs. the normal hydrogen electrode (NHE). As seen in Figure 1, the anodic current increases when the potential of water oxidation covers the delithiation potential found in organic solvent corresponding to $\approx 1.5 \mathrm{~V}$ vs. NHE. For $\mathrm{La}_{2} \mathrm{LiRuO}_{6}$, the oxidation in acidic conditions is non-reversible and the current fades with cycling (Supplementary Figure 3), typical of the dissolution of the highly soluble $\mathrm{RuO}_{4}{ }^{-}$species. ${ }^{19,34,35}$ Therefore this catalyst won't be further studied. For the Ir-based material, the current is reversible and stable for 50 cycles with no bulk modifications as observed by XRD (Supplementary Figure 4). A redox peak at $1.5 \mathrm{~V}$ vs. NHE can be seen for LaLilrO 6 in the pseudocapacitive region (Supplementary Figure 5), corresponding to the delithiation potential measured in organic solvent. This singular behavior is in contrast with the stateof-the-art $\mathrm{IrO}_{2}$ catalyst (and $\mathrm{RuO}_{2}$ ) for which the OER activity measured in acidic and alkaline conditions is almost identical (higher in acidic conditions by a factor of $\approx 3-4$ ). ${ }^{23}$ At first sight, one could explain this trend by the fact that increasing the metal oxidation state from $\mathrm{Ir}^{4+}$ in $\mathrm{IrO}_{2}$ to $\mathrm{Ir}^{5+}$ in $\mathrm{La}_{2} \mathrm{LilrO}_{6}$ decreases the iridium chemical potential, making the $\mathrm{Ir}-\mathrm{O}$ bond more covalent and therefore weaker in energy. Within this scenario, the oxygen binding strength on the surface of $\mathrm{La}_{2} \mathrm{LilrO}_{6}$ would fall into the weak side of the volcano plot. ${ }^{8,12}$. Nevertheless, when positioning the $\mathrm{Ir}^{6+} / \mathrm{Ir}^{5+}$ and $\mathrm{Ir}^{5+} / \mathrm{Ir}^{4+}$ redox potential found for $\mathrm{La}_{2} \mathrm{LiIrO}_{6}$ and $\mathrm{IrO}_{2}{ }^{22,24}$ respectively, with the oxygen standard potential $\mathrm{E}^{\circ}\left(\mathrm{O}_{2} / \mathrm{H}_{2} \mathrm{O}\right)$ it clearly appears that, overall, a key feature for these iridium oxides surfaces to be active is their ability to be oxidized(Fig. $1 \mathrm{C})$. Indeed, while the surface of $\mathrm{IrO}_{2}$ is oxidized in both alkaline and acidic conditions, ${ }^{24}$ acidic conditions are required to allow for the surface oxidation and delithiation of $\mathrm{La}_{2} \mathrm{LilO}_{6}$. This is reinforced by the enhanced OER activity measured in alkaline conditions after activation of the surface in acidic conditions (Supplementary Figure 6) as well as the crystalline surface found after cycling at pH 13 (Supplementary Figure 7).

Figures $2 \mathrm{a}$ and $2 \mathrm{~b}$ show the OER activity for $\mathrm{La}_{2} \mathrm{LilOO}_{6}$ normalized by weight and per surface area compared to that of $7 \mathrm{~nm}$-size $\mathrm{IrO}_{2}$ particles reported elsewhere. ${ }^{23}$ While the weight normalized 
activities are similar, the OER activity normalized by surface area for $\mathrm{La}_{2} \mathrm{LillO}_{6}$ at $\mathrm{pH} 1$ is ca. 50 times larger than both nanometric ${ }^{23}$ and micrometric commercial $\mathrm{IrO}_{2}$ and ranks among the most active Irand Ru-based oxides reported so far (Supplementary Figure 8). To further understand the large surface activity found for $\mathrm{La}_{2} \mathrm{LilO}_{6}$, the evolution of the pseudocapacitive charge $\mathrm{q}^{*}$ was tracked down over 50 cycles (Fig. $2 c$ and 2 d). The pseudocapacitive charge $\mathrm{q}^{*}$, previously shown to be correlated with the amount of surface iridium active sites and therefore to the OER activity, ${ }^{22,36}$ displays an exponential increase with the value reaching a maximum after 20 cycles (Fig. 2d). Its evolution does not follow the OER activity which remains stable from the first to the $50^{\text {th }}$ cycle (Fig. $2 d)$, suggesting that the surface modifications are not responsible for the large OER activity and occur on the surface of $\mathrm{La}_{2} \mathrm{LilrO}_{6}$ during the initial cycles which then stabilizes.

\section{Surface oxidation and reconstruction}

Using high-resolution TEM (HRTEM), the increase of the pseudocapacitive charge $q^{*}$ is explained by the gradual formation of $\mathrm{IrO}_{2}$ nanoparticles on the surface of $\mathrm{La}_{2} \mathrm{LilrO}_{6}$ particles. The [201] oriented HRTEM image of the pristine sample (Fig. 3a) shows that a perfectly ordered perovskite structure with the space group $\mathrm{P}_{1} / \mathrm{C}$ corresponding to $\mathrm{La}_{2} \mathrm{LilrO}_{6}$ persists throughout the sample, including the surface (Fig. 3b). Similarly, the HRTEM image viewed along the [201] direction for $\mathrm{La}_{2} \mathrm{LillO}_{6}$ after 1 cycle at pH 1 shows a similar ordered structure (Fig. 3c) with a surface remaining equally crystalline

(Fig. 3d). Bearing in mind that the OER activity remains constant from the first to the $50^{\text {th }}$ cycle, this observation definitively rules out the formation of secondary phases to explain the large OER activity found for $\mathrm{La}_{2} \mathrm{LilrO}_{6}$ at $\mathrm{pH} 1$. After 25 cycles, the same basic reflections corresponding to $\mathrm{La}_{2} \mathrm{LilrO}_{6}$ are observed (Fig. 3e). Moreover, a random distribution of local bright and dark zones (or channels) is observed and accompanied with the formation of nanoparticles on the surface of the electrode with a mean diameter of $3.5 \mathrm{~nm}$ (Supplementary Figure 9) that were identified as $\mathrm{IrO}_{2}$ (Fig. 3f). After 50 cycles, the FFT shows the emergence of extra reflections which are associated with the ordering observed along the [201] zone axis in the HRTEM image (white arrows in Fig. 3g) and the formation 
of a superstructure (Supplementary Figure 10). The simultaneous growth of $\mathrm{IrO}_{2}$ particles on the surface upon cycling to reach $6.5 \mathrm{~nm}$ in diameter (Fig. 3h and Supplementary Figure 9) together with the gradual ordering of defects within the bulk of $\mathrm{La}_{2} \mathrm{LilrO}_{6}$ suggest a migration of Ir from the bulk to the surface, presumably triggered by the Li migration that creates vacant sites for Ir to diffuse. This observation, combined with the OER activity which is independent on the pseudocapacitive charge $\mathrm{q}^{*}$ site, shines light on a complex oxidation mechanism enlisting both migration as well as surface reorganization.

To grasp further information on the oxidation process happening during the oxidation, we combined complementary differential electrochemical mass spectrometry (DEMS) measurements and density functional theory (DFT) calculations. The DEMS measurement was carried out in organic solvent to detect oxygen from the lattice upon oxidation independently from oxygen generated from water oxidation (Supplementary Figure 11). Starting from 4.5 V vs. $\mathrm{Li}^{+} / \mathrm{Li}$, gaseous oxygen is detected but in a very limited amount $(\approx 1.5 \%$ of the total charge) indicative of a surface oxygen loss process. In addition to molecular oxygen, $\mathrm{CO}_{2(\mathrm{~g})}$ is simultaneously detected during the oxidation, indicative of a reactive surface towards the decomposition of carbonates. Figure 4 shows the atom-projected density of states (pDOS) computed from DFT for the pristine and the delithiated phases. The pDOS clearly evidence a strong oxygen character of the band lying just below the Fermi level (Fig. 4a and $4 d)$. In the delithiated phase, the $O(p)$ contribution is even higher than the $\operatorname{Ir}(d)$ contribution due to the electrostatic destabilization of the p-band induced by Li removal. ${ }^{26}$ When plotting the crystal orbital overlap populations (COOPs), the Ir-O COOPs are mostly bonding below the Fermi level (COOPs $>0)$ and antibonding around the Fermi level (COOPs $<0)$ for the pristine phase (Fig. 4b), revealing that the dominant contribution of the O-states at the Fermi level in the delithiated $\mathrm{La}_{2} \mid \mathrm{rO}_{6}$ arises from a p/d-band overlap. The positive Fukui function (Fig. 4c) shows that the electronic levels involved in the delithiation of $\mathrm{La}_{2} \mathrm{LillO}_{6}$ are $\mathrm{t}_{2 \mathrm{~g}}$-like local levels. At $\mathrm{pH}=13$ where the delithiation process does not take place, these orbitals do not provide a reactive site for the OER. This confirms a previous belief that $\mathrm{e}_{\mathrm{g}}$-like $\sigma^{*}$ orbitals are the active site for the OER reaction on the surface of 
$\operatorname{LaM}(3 \mathrm{~d}) \mathrm{O}_{3}$ perovskites in alkaline solution. ${ }^{8,13}$ For the delithiated $\mathrm{La}_{2} \mid \mathrm{rO}_{6}$ (Fig. $4 \mathrm{~d}$ ), two oxygen $p$ orbitals having no overlap with the $\operatorname{Ir}(d)$-orbitals also occur in the Fukui function in addition to the $\mathrm{t}_{2 \mathrm{~g}^{-}}$ like $\pi^{*}$ orbitals, which confirms the activation of pure O-states upon delithiation. In order to stabilize these unstable O-holes under further oxidizing conditions (OER conditions), structural and charge reorganization on the surface is expected. Two different scenarios can be envisioned to achieve such surface stabilization with either the direct coupling of two surface oxygens or the reaction of surface oxygen with a nucleophilic species such as carbonate or water. Both of these mechanisms have been discussed for molecular OER catalysts. ${ }^{14}$ Following the results from DEMS measurements, both mechanisms are likely to be at play on the surface of the delithiated $\mathrm{La}_{2} \mid \mathrm{IO}_{6}$ compound, but with the direct coupling probably being limited.

In addition to the oxygen reactivity, STEM-EDX mapping performed on a sample cycled 50 times shows that the surface of $\mathrm{La}_{2} \mathrm{IrO}_{6}$ is poor in lanthanum and rich in iridium (Supplementary Figures 12 and 13), and this was further confirmed by XPS analysis (Supplementary Figure 14 and Table 3). This observation indicates that La- and Li-deficient surfaces are unstable and reconstruct, thus explaining the growth of $\mathrm{IrO}_{2}$ nanoparticles on the surface. However, this process is limited since the bulk structure remains crystalline after 50 cycles and the $\mathrm{IrO}_{2}$ nanoparticles only form on the top $20 \mathrm{~nm}$ of the surface where the $\mathrm{La}^{3+}$ deficiency was observed. Moreover, the charge current passed after 50 cycles is two orders of magnitude greater than the one required to form $\mathrm{IrO}_{2}$ from the pristine phase (Supplementary Figure15). This implies the onset of a side reaction arising from the large reactivity of surface oxygen upon oxidation. Interestingly, at the surface of $\mathrm{La}_{2} \mathrm{IrO}_{6}$ the atomic Bader charges show that some oxygen atoms become more electrophilic (oxidized compared to bulk Fig. $4 \mathrm{~g}$ ) and can go through a nucleophilic attack from the water lone-pairs, hence enabling the OER. Concomitantly, other surface oxygen atoms become more nucleophilic (reduced compared to the bulk) and can be protonated.

\section{Atomic migration}


The oxidation mechanism described above is further supported by observing the lattice modifications encountered after cycling via HRTEM. The pristine phase shows a perfect periodic arrangement of cations (Fig. 5a). This drastically contrast with the pattern collected after 50 cycles which is largely modified with an intensity alternation of atomic columns corresponding to a variation of atomic density observed in a periodic fashion (Fig. $5 b$ and $5 c$ ) as well as a strong compression and extension of cationic distances from $3.4 \AA$ in the pristine phase to $3.0 \AA$ and $3.7 \AA$ (Fig. 5b). HRTEM images were then simulated for different values of Ir atom occupancy. The best match between the HRTEM images and simulation was obtained for a combination of columns along the migration direction with a successive Iridium occupancy of $0 \%, 50 \%$ and $100 \%$ every two atomic lines (Fig. $5 \mathrm{~d}$ and Supplementary Figure 16). Since the modification of the relative intensities from row-to-row excludes any effect due to thickness variation, the gradual decrease of the atomic intensity from the bulk to the surface further confirms that migration occurs to counterbalance the atomic loss on the surface during the oxidation process (Supplementary Figure 16). More striking are the black spots forming hexagonal shapes found in the filtered HRTEM image in Fig. $5 \mathrm{c}$ and reproduced in Fig $5 \mathrm{~d}$ and simulated in Fig $5 e$ and $5 f$. Even though the physical origin for the formation of these hexagons remains to be fully understood (see simulations in Supplementary Information Figure 17), they suggest a complex cationic and anionic migration process which is associated with the consumption of both iridium and oxygen on the surface of the catalyst, as found by HRTEM and DEMS measurements, respectively. Importantly, this phenomenon leads to the modification of the Ir coordination on the surface and its dissolution as detected by ICP (Supplementary Table 4), explaining the relatively low stability previously reported for Ir- or Ru-based oxides that exhibit enhanced OER activity. ${ }^{18,20,21}$ The surface modification combined with the applied voltage creates a driving force for oxygen to migrate from the bulk to the surface for replenishing the oxygen vacancies created during the OER. Finally, it is worth noting that the strains arising from these drastic structural rearrangements are eventually released by the creation of a large concentration of dislocations in the bulk of the delithiated phase (Fig. $5 \mathrm{~g}$ ). This effect is most likely rooted in the lower bulk modulus 
calculated for the delithiated $\mathrm{La}_{2} \mid \mathrm{IO}_{6}$ phase compared to the $\mathrm{IrO}_{2}$ particles formed on the surface (Supplementary Figure 18).

\section{Overall OER mechanism}

To complete the OER mechanism, X-ray absorption (XAS) measurements at the $\mathrm{Ir} \mathrm{L}_{\mathrm{III}}$-edge combined with the determination of the $\mathrm{H}^{+}$reaction order by electrochemical measurements were performed. The surface Ir oxidation state has been measured by XAS in the total electron yield (TEY) mode after oxidation at $1.6 \mathrm{~V}$ vs. reversible hydrogen electrode (RHE) and was compared to the pristine sample (Fig. 6a and 6b). While the pristine sample clearly indicates $\operatorname{~r~}^{5+}$ oxidation state, a shift to lower energy corresponding to a reduction of iridium at an oxidation state close to $4+$ is clearly observed after oxidation at $1.6 \mathrm{~V}$ vs. RHE. At first, this reduction under oxidative conditions could be seen as counter-intuitive. However, it is well-explained by the fact that for the oxidized phase, $\mathrm{O}_{2(\mathrm{~g})}$ is lost on the surface as shown by combining DEMS measurements and DFT calculations, leading to the reduction of the metal oxidation state following the equation: $\operatorname{lr}^{n+} O_{x} \rightarrow \mid r^{(n-2 \delta)+} O_{x-\delta}+\delta / 2 O_{2(g)}+2 \delta e^{-} \cdot A$ similar behavior has been measured for $\mathrm{IrO}_{2}$ in acidic conditions, ${ }^{37}$ known to lose oxygen under OER conditions. ${ }^{27}$ This observation is further confirmed by XPS where a shift of the $\operatorname{Ir} 4 \mathrm{f}$ peak to lower binding energy was observed after cycling (Supplementary Figure 14). Regarding the $\mathrm{H}^{+}$reaction order, a Tafel slope of $50 \mathrm{mV} /$ decade is observed at pH 1 (Fig. 6c) and a pH dependence of around 40 $\mathrm{mV} /$ decade is found (Fig. $6 \mathrm{~d}$ ), giving a reaction order towards $\mathrm{H}^{+}$of 0.87 on the SHE scale and -0.31 on the RHE scale. ${ }^{38}$ This slight deviation from 1 on the SHE scale and 0 on the RHE scale, values normally associated with a concerted proton-electron transfer, could indicate a pH dependence and a sequential proton and electron transfer. ${ }^{39}$ However, as discussed for hydrous $\mathrm{IrO}_{2}{ }^{24}$ this deviation most probably arises from proton mobility between different surface oxygen groups. We postulate that while some of the oxidized surface oxygens act as water adsorption sites as discussed previously, the reduced oxygen atoms act as proton acceptors during the reaction of water with the electrophilic oxygens. 
The complete OER mechanism which enlists several steps is summarized in Fig. 6e. The first activation step consists of oxidizing $\mathrm{Ir}^{5+}$ to $\mathrm{Ir}^{6+}$ by taking an electron from $\mathrm{t}_{2 \mathrm{~g}}$-like $\pi^{*}$ states which have been shown to be inactive for OER. For $\mathrm{La}_{2} \mathrm{LilrO}_{6}$, this first oxidation can only be achieved in acidic conditions for which the delithiation potential can be reached (step 1 in Fig. 6e, this oxidation is nonreversible as shown by the OER activity being independent on the Li+ concentration, Supplementary Figure 20). In contrast, for $\mathrm{IrO}_{2}$ this surface oxidation can also be achieved in alkaline conditions. When further oxidizing the surface under OER conditions, purely oxygen states are oxidized, creating oxygen radicals (electrophilic oxygen) on the surface. These surface oxygen atoms can be stabilized by either reacting with water through nucleophilic attack from the water oxygen lone pairs followed by deprotonation via a CPET mechanism (step 2) or by forming an O-O bond by sharing its ligand holes with another surface oxygen (step 3). However, based on the relative stability of the bulk of $\mathrm{La}_{2} \mathrm{IrO}_{6}$ observed by XRD and HRTEM after multiple cycles, we postulate that step 2 is the most predominant one and is the responsible for the large OER activity. Eventually, the surface O-O species form molecular dioxygen, leaving oxygen vacancies on the surface of the oxide and reducing the Ir oxidation state. Then, these vacancies can be filled by the migration of bulk oxygen to the surface (step 4). However, the kinetics associated with the bulk oxygen migration is most probably slow when compared to the kinetics associated with the filling of the oxygen vacancies by water (step 5). This explains that the bulk structure is conserved even after 50 cycles and only the top few nanometers undergo a drastic modification.

\section{Conclusions}

In summary, using $\mathrm{La}_{2} \mathrm{LilrO}_{6}$ as a model catalyst we were able to demonstrate that the $\mathrm{Ir}^{5+}$-O bond is not active for OER. The formation of the oxidized surface at $\mathrm{pH} 1$ leads to an enhanced OER activity as the Fermi level enters the non-bonding pure oxygen states for $\mathrm{La}_{2} \mathrm{IO}_{6}$ under oxidative conditions, creating oxygen radicals on the surface which behave as electrophilic centers prone to nucleophilic attack from the water oxygen lone pairs. This demonstrates that $t_{2 \mathrm{~g}}$-like $\pi^{*}$ orbitals are not active for 
OER and that the large activity often encountered for oxidized Ir-based surfaces is rooted in the oxidation of pure oxygen states, explaining the loss of oxygen (reductive elimination) previously observed. ${ }^{27,37}$ We also demonstrate that drastic structural reorganization is experienced when triggering the reactivity of surface oxygen, with cationic migration occurring from the bulk to the surface. More importantly, this work provides the fundamental understanding to explain previous reports demonstrating that the OER activity for Ir- or Ru-based oxides is often associated with surface instability. ${ }^{18-21}$ Our results demonstrate that this correlation is rooted in the activation of surface oxygen which leads to the modification of the Ir coordination. This finding suggests that new OER catalysts can be made highly active through the oxidation of their surface, providing that such highly reactive surfaces can be stabilised through the use of an appropriate synthesis route. However, further work will be necessary to fully understand if this type of oxides is suitable as OER catalysts for long term application. Finally, the demonstration of surface reconstruction and atomic migration triggered by the reactivity of the electrophilic oxidized surface oxygen with a nucleophile solvent molecule will serve the field of Li-ion batteries where positive electrodes such as Li-rich layered compounds exhibit the same oxygen oxidation ${ }^{25,40-42}$ and surface reactivity process. ${ }^{43,44}$

\section{Methods}

\section{Materials synthesis and characterizations}

$\mathrm{La}_{2} \mathrm{LillO}_{6}$ and $\mathrm{La}_{2} \mathrm{LiRuO}_{6}$ were synthesized by solid-state reaction of precursors $\mathrm{La}_{2} \mathrm{O}_{3}$ (Alfa Aesar 99.99\%) previously dried at $1000^{\circ} \mathrm{C}$ overnight, $\mathrm{Li}_{2} \mathrm{CO}_{3}$ (Sigma Aldrich > 99\%), IrO 2 (Alfa Aesar 99\%) and $\mathrm{RuO}_{2}$ (Alfa Aesar $99.9 \%$ ), respectively, and fired at $900^{\circ} \mathrm{C}$ under $\mathrm{O}_{2}$ for $24 \mathrm{~h}$ with intermediate grinding for $\mathrm{La}_{2} \mathrm{LilO}_{6}$ and $980^{\circ} \mathrm{C}$ for $24 \mathrm{~h}$ with intermediate grinding for $\mathrm{La}_{2} \mathrm{LiRuO}_{6}$. X-ray powder diffraction (XRD) patterns were recorded using a BRUKER D8 Advance diffractometer using Cu-Ka radiation. The crystallographic parameters were determined by Le Bail method using FullProf suite. 


\section{Electrochemical study}

Electrochemical studies in organic solvent were carried out in Swagelok type cells with Li metal counter electrode and LP30 electrolyte (BASF) which were assembled in an argon filled glove box. The active material was ball milled with $20 \%$ wt carbon black (Super P, Timcal) using SPEX ball mill for 20 minutes. Galvanostatic cycling was performed at $\mathrm{C} / 10$.

The amount of oxygen and carbon dioxide released during electrochemical cycling was obtained using differential electrochemical mass spectrometry (DEMS). The configuration has been described elsewhere while the electrode preparation method and experimental details are included elsewhere. The cell was cycled in galvanostatic mode at $\mathrm{C} / 10$ in a potential with a cut-off of $5 \mathrm{~V} \mathrm{vs.} \mathrm{Li}^{+} / \mathrm{Li}$ with the gas evolved being probed in 15 min intervals.

Electrochemical measurements in aqueous solution were carried by drop casting an ink made by mixing THF (Sigma-Aldrich 99.9\%) with the perovskite oxide powder and ethylene black carbon (Alfa Aesar $99.9 \%$ ) in a 5:1 weight ratio as well as Nafion binder (5\% weight, lon power), following the recipe described elsewhere. ${ }^{45}$ Note that for measurements in alkaline solution, the Nafion was neutralized by $\mathrm{KOH}$ while at neutral and high $\mathrm{pH}$ the Nafion was used as received. Glassy carbon electrodes with a diameter of $0.196 \mathrm{~cm}^{2}$ were used as support with a loading of active material of 50 $\mu \mathrm{g}$ per electrode. Rotating disk electrode measurements were performed in glass cell previously cleaned by acid treatment followed by boiling in water for 2 hours. PINE Instrument rotating device was used at $1600 \mathrm{rpm}$. Solution of $0.1 \mathrm{M} \mathrm{KOH}$ was used for $\mathrm{pH}=13$. For measurements in acid, $\mathrm{H}_{2} \mathrm{SO}_{4}$ was used at different concentrations. For measurements in neutral $\mathrm{pH}$, a $0.1 \mathrm{M} \mathrm{H}_{2} \mathrm{SO}_{4}$ solution was made, $1 \mathrm{M} \mathrm{KNO}_{3}$ was added to keep a large ionic strength, and the solution was adjusted to $\mathrm{pH} 7$ by adding $\mathrm{KOH}$ pellets. All those measurements were carried out in $\mathrm{O}_{2}$-saturated solutions. To prepare XRD samples, gas diffusion layer was used as a support and large loading of $500 \mu \mathrm{g}$ per electrode was used. The powder was collected after being washed by ethanol and sonicated. To prepare XAS and TEM samples, large glassy carbon electrode with a surface of $1 \mathrm{~cm}^{2}$ was used, with a loading of $1 \mathrm{mg}$ 
per electrode. Hence, the OER activity measured for those electrodes differs from the one measured when using the optimized $0.196 \mathrm{~cm}^{2}$ glassy carbon electrode with $50 \mu \mathrm{g}$ loading.

We deliberately chose to normalize the current density by weight in order to compare the catalysts studied in the manuscript. This choice is motivated by the misrepresentation any normalization by specific surface area obtained on the pristine sample would induce as the surface is greatly modified during the activation process. This also allows for a direct visualization of the large activity obtained with non-optimized morphologies and the great impact such materials would have after optimization.

\section{Transmission electron microscopy}

High-resolution TEM and EDX-STEM experiments were acquired using a FEI-TITAN 80-300 kV with SuperX EDX detector (Cs probe corrector and X-FEG), a FEI-TITAN 80-300 kV (Cs image corrector) and a Tecnai G2 F20ST 200 kV. HR-TEM images were calculated using both the Dr. Probe software ${ }^{46}$ and the MactempasX version 2 software. Fast Fourier transform (FFT) filters were applied using FIJI software for the visualization of dislocation and to reduce the level of noise of the high-resolution images with circle masks (diameters of $8-10 \mathrm{~nm}^{-1}$ ) surrounding each bright spots.

\section{Density functional theory calculations}

Spin-polarized density functional theory (DFT) calculations were performed using the plane-wave density functional theory VASP (Vienna Ab initio Simulation Package) code ${ }^{47,48}$ within the generalized gradient approximation of Perdew-Burke-Ernzerhof (PBE) to describe electron exchange and correlation. ${ }^{49}$ The rotationally invariant Dudarev method (DFT+U) was used to correct the selfinteraction error of conventional DFT for correlated d-electrons. ${ }^{50}$ Different $U$ parameters varying from 0 to $4 \mathrm{eV}$ for Ru and Ir as well as spin-orbit coupling were tested which led to slightly different crystal structures, DOS and COOP but did not affect the trend of the redox mechanism presented in this paper. All atom coordinates and lattice parameters were fully relaxed using conjugate gradient 
energy minimization until the forces acting on each atom were less than $5.10^{-3} \mathrm{eV} . \AA^{-2}$. A plane-wave cutoff of $600 \mathrm{eV}$ was used to define the basis set, with a $(6 \times 6 \times 4)$ well-converged k-point sampling for each compound. The COOPs were computed using the Lobster program developed by Dronskowski and coworkers. ${ }^{51-53}$ The Fukui functions are computed as the difference between the electron density of the neutral system and the oxidized system (ca. $2 \cdot 10^{-3}$ electron removed).

\section{X-ray absorption spectroscopy at the $\mathrm{Ir}_{\text {III }}$ L-edge}

Samples for XAS measurements were prepared by loading 500mg onto a large glassy carbon plate using the same ink composition as described above. The potential was held for $10 \mathrm{~min}$ at $1.6 \mathrm{~V}$ vs. RHE in $0.1 \mathrm{M} \mathrm{H}_{2} \mathrm{SO}_{4}$ solution, using $\mathrm{Ag} / \mathrm{AgCl}$ as reference and $\mathrm{Pt}$ as counter-electrode. No rotation was applied. The potential was kept when removing the sample from the electrolyte. The XAS measurements were carried out at DIAMOND on the total electron yield mode. The oxidized sample was compared to a pristine sample as well as commercial $\mathrm{IrO}_{2}$ (Aldrich) that was used as received.

\section{Data availability statements}

The data that support the plots within this paper and other findings of this study are available from the corresponding author upon reasonable request

\section{Additional information}

Supplementary information is available in the online version of the paper. Correspondence and requests for materials should be addressed to A.G.

\section{Competing financial interests}

The authors declare no competing financial interests.

\section{Acknowledgments}

We thank Juri Barthel of Ernst Ruska-Centre (Forschungszentrum Jülich) for valuable discussions and his support for the HRTEM simulation using Dr Probe software. We would like to thank Domitille 
Giaume (ENSCP) for the ICP measurements and Dominique Foix (IPREM) for the XPS measurements. We would also like to thank Erik Berg (PSI) for the DEMS measurements. We acknowledge Diamond Light Source for time awarded to the Energy Materials BAG on Beamline B18, under proposal sp12559 as well as Alan Chadwick and Giannantonio Cibin for assistance and discussions. The authors acknowledge financial support from the European Union under the $7^{\text {th }}$ Framework Program under a contract for an Integrated Infrastructure Initiative reference 312483-ESTEEM2. We acknowledge funding from the European Research Council (ERC) (FP/2014)/ERC Grant-Project 670116-ARPEMA.

\section{Author contributions}

A.G. designed the experiments. A.G. made the synthesis, the structural and electrochemical analysis. A.D., W.D. and M.D. performed TEM measurements, atomic structural analyses and HRTEM image simulations. M.S. and M.-L.D. carried out the DFT calculations. A.G. and J.-M.T. wrote the manuscript and all authors edited the manuscript.

\section{References}

1. Dau, H. et al. The Mechanism of Water Oxidation: From Electrolysis via Homogeneous to Biological Catalysis. ChemCatChem 2, 724-761 (2010).

2. Hong, W. et al. Toward the rational design of non-precious transition metal oxides for oxygen electrocatalysis. Energy Environ. Sci. 8, 1404-1427 (2015).

3. Matsumoto, Y. \& Sato, E. Electrocatalytic properties of transition metal oxides for oxygen evolution reaction. Mater. Chem. Phys. 14, 397-426 (1986).

4. Wattiaux, a. Electrolytic Oxygen Evolution in Alkaline Medium on La[sub 1-x]Sr[sub x] FeO[sub 3-y] Perovskite-Related Ferrites. J. Electrochem. Soc. 134, 1718 (1987).

5. Bockris, J. O. \& Otagawa, T. The electrocatalysis of oxygen evolution on perovskites. J Electrochem Soc 131, 290-302 (1984).

6. Yagi, S. et al. Covalency-reinforced oxygen evolution reaction catalyst. Nat. Commun. 6, 8249 (2015). 
7. Hardin, W. G. et al. Tuning the Electrocatalytic Activity of Perovskites through Active Site Variation and Support Interactions. Chem. Mater. 26, 3368-3376 (2014).

8. Suntivich, J., May, K. J., Gasteiger, H. a, Goodenough, J. B. \& Shao-Horn, Y. A perovskite oxide optimized for oxygen evolution catalysis from molecular orbital principles. Science 334, 13835 (2011).

9. Grimaud, A. et al. Double perovskites as a family of highly active catalysts for oxygen evolution in alkaline solution. Nat. Commun. 4, 2439 (2013).

10. Calle-Vallejo, F. et al. Number of outer electrons as descriptor for adsorption processes on transition metals and their oxides. Chem. Sci. 4, 1245 (2013).

11. Trasatti, S. Electrocatalysis by oxides - Attempt at a unifying approach. J. Electroanal. Chem. 111, 125-131 (1980).

12. Man, I. C. et al. Universality in Oxygen Evolution Electrocatalysis on Oxide Surfaces. ChemCatChem 3, 1159-1165 (2011).

13. Matsumoto, Y., Manabe, H. \& Sato, E. Oxygen Evolution on Lal_ Sr CoO Electrodes in Alkaline Solutions. J. Electrochem. Soc. 127, 811-814 (1980).

14. Betley, T. A., Wu, Q., Voorhis, T. Van \& Nocera, D. G. Electronic design criteria for O-O bond formation via metal-oxo complexes. Inorg. Chem. 47, 1849-1861 (2008).

15. Risch, M. et al. Structural Changes of Cobalt-Based Perovskites upon Water Oxidation Investigated by EXAFS. J. Phys. Chem. C 117, 8628-8635 (2013).

16. May, K. J. et al. Influence of oxygen evolution during water oxidation on the surface of perovskite oxide catalysts. J. Phys. Chem. Lett. 3, 3264-3270 (2012).

17. Binninger, T. et al. Thermodynamic explanation of the universal correlation between oxygen evolution activity and corrosion of oxide catalysts. Sci. Rep. 5, 12167 (2015).

18. Danilovic, N. et al. Using surface segregation to design stable Ru-Ir oxides for the oxygen evolution reaction in acidic environments. Angew. Chem. Int. Ed. Engl. 53, 14016-21 (2014).

19. Chang, S. H. et al. Functional links between stability and reactivity of strontium ruthenate single crystals during oxygen evolution. Nat. Commun. 5, 4191 (2014).

20. Reier, T. et al. Molecular Insight in Structure and Activity of Highly Efficient, Low-Ir Ir-Ni Oxide Catalysts for Electrochemical Water Splitting (OER). J. Am. Chem. Soc. 137, 13031-40 (2015).

21. Cherevko, S. et al. Oxygen and hydrogen evolution reactions on $\mathrm{Ru}, \mathrm{RuO} 2$, Ir, and IrO2 thin film electrodes in acidic and alkaline electrolytes: A comparative study on activity and stability. Catal. Today 262, 170-180 (2016).

22. Stoerzinger, K. A., Qiao, L., Biegalski, M. D. \& Shao-horn, Y. Orientation-Dependent Oxygen Evolution Activities of Rutile IrO 2 and RuO2. J. Phys. Chem. Lett. 118, 19733-19741 (2014). 
23. Lee, Y., Suntivich, J., May, K. J., Perry, E. E. \& Shao-horn, Y. Synthesis and Activities of Rutile IrO 2 and RuO 2 Nanoparticles for Oxygen Evolution in Acid and Alkaline Solutions. (2012).

24. Lyons, M. E. G. \& Floquet, S. Mechanism of oxygen reactions at porous oxide electrodes. Part 2--Oxygen evolution at $\mathrm{RuO} 2, \operatorname{IrO} 2$ and $\operatorname{Ir}(\mathrm{x}) \mathrm{Ru}(1-\mathrm{x}) \mathrm{O} 2$ electrodes in aqueous acid and alkaline solution. Phys. Chem. Chem. Phys. 13, 5314-35 (2011).

25. Grimaud, A., Hong, W. T., Shao-Horn, Y. \& Tarascon, J.-M. Anionic redox processes for electrochemical devices. Nat. Mater. 15, 121-126 (2016).

26. Saubanère, M., McCalla, E., Tarascon, J.-M. \& Doublet, M.-L. The intriguing question of anionic redox in high-energy density cathodes for Li-ion batteries. Energy Environ. Sci. 9, 984-991 (2016).

27. Fierro, S., Nagel, T., Baltruschat, H. \& Comninellis, C. Investigation of the oxygen evolution reaction on $\mathrm{Ti} / \mathrm{IrO} 2$ electrodes using isotope labelling and on-line mass spectrometry. Electrochem. commun. 9, 1969-1974 (2007).

28. Wohlfahrt-Mehrens, M. \& Heitbaum, J. Oxygen evolution on Ru and RuO2 electrodes studied using isotope labelling and on-line mass spectrometry. J. Electroanal. Chem. 237, 251-260 (1987).

29. Surendranath, Y., Kanan, M. W. \& Nocera, D. G. Mechanistic Studies of the Oxygen Evolution Reaction by a Cobalt-Phosphate Catalyst at Neutral pH. 16501-16509 (2010). doi:10.1021/ja908730h.(45)

30. Graeupner, J. et al. Probing the Viability of Oxo-Coupling Pathways in Iridium-Catalyzed Oxygen Evolution. Organometallics 32, 5384-5390 (2013).

31. Rong, X., Parolin, J. \& Kolpak, A. M. A Fundamental Relationship between Reaction Mechanism and Stability in Metal Oxide Catalysts for Oxygen Evolution. ACS Catal. 6, 11531158 (2016).

32. Jung, D. Y., Demazeau, G. \& Choy, J. H. Preparation under oxygen pressures of new perovskites: (ALa)LilrO 6- $\delta(\mathrm{A}=\mathrm{Ca}, \mathrm{Sr}, \mathrm{Ba})$. High Press. Res. 15, 121-125 (1996).

33. Hayashi, K., Demazeau, G., Pouchard, M. \& Hagenmuller, P. Preparation and magnetic study of a new iridium (V) perovskite: LaLiO.5IrO.503. Mater. Res. Bull. 15, 461-467 (1980).

34. Cherevko, S. et al. Dissolution of Noble Metals during Oxygen Evolution in Acidic Media. ChemCatChem 6, 2219-2223 (2014).

35. Hodnik, N. et al. New Insights into Corrosion of Ruthenium and Ruthenium Oxide Nanoparticles in Acidic Media. J. Phys. Chem. C 119, 10140-10147 (2015).

36. Burke, L. D. \& Murphy, O. J. Cyclic voltammetry as a technique for determining the surface area of RuO2 electrodes. J. Electroanal. Chem. 96, 19-27 (1979).

37. Minguzzi, A. et al. Observing the oxidation state turnover in heterogeneous iridium-based water oxidation catalysts. Chem. Sci. 5, 3591 (2014). 
38. Giordano, L. et al. pH dependence of OER activity of oxides: Current and future perspectives. Catal. Today (2015). doi:10.1016/j.cattod.2015.10.006

39. Koper, M. T. M. Theory of multiple proton-electron transfer reactions and its implications for electrocatalysis. Chem. Sci. 4, 2710 (2013).

40. Aydinol, M. K., Kohan, A. F. \& Ceder, G. Ab initio study of lithium intercalation in metal oxides and metal dichalcogenides. Phys. Rev. B 56, 1354-1365 (1997).

41. Sathiya, M. et al. Reversible anionic redox chemistry in high-capacity layered-oxide electrodes. Nat. Mater. 12, 827-35 (2013).

42. McCalla, E. et al. Visualization and impact of O-O peroxo-like dimers in high capacity layered oxides for Li-ion batteries. Science (80-. ). 350, 1516-1521 (2015).

43. Yabuuchi, N., Yoshii, K., Myung, S., Nakai, I. \& Komaba, S. Detailed Studies of a High-Capacity Electrode Material for rechargeable batteries, Li2MnO3-LiCo1/3Ni1/3Mn1/3O2. J. Am. Ceram. Soc. 133, 4404-4419 (2011).

44. Castel, E., Berg, E. J., Kazzi, M. El, Nova, P. \& Villevieille, C. Di ff erential Electrochemical Mass Spectrometry Study of the Interface of $x \operatorname{Li} 2 \mathrm{MnO} 3 \cdot(1-\mathrm{x}) \operatorname{LiMO} 2(\mathrm{M}=\mathrm{Ni}, \mathrm{Co}$, and $\mathrm{Mn}$ ) Material as a Positive Electrode in Li-Ion Batteries. Chem. Mater. 26, 5051-5057 (2014).

45. Suntivich, J., Gasteiger, H. A., Yabuuchi, N. \& Shao-Horn, Y. Electrocatalytic Measurement Methodology of Oxide Catalysts Using a Thin-Film Rotating Disk Electrode. J. Electrochem. Soc. 157, B1263-B1268 (2010).

46. Barthel, J. Dr. Probe - High-resolution (S)TEM image simulation software. http://www.erc.org/barthel/drprobe/ (2016).

47. Kresse, G. \& Furthmüller, J. Efficient iterative schemes for ab initio total-energy calculations using a plane-wave basis set. Phys. Rev. B - Condens. Matter Mater. Phys. 54, 11169-11186 (1996).

48. Kresse, G. \& Hafner, J. Ab initio molecular dynamics for liquid metals. Phys. Rev. B 47, 558561 (1993).

49. Perdew, J. P. \& Wang, Y. Accurate and simple analytic representation of the electron-gas correlation energy. Phys. Rev. B 45, 13244-13249 (1992).

50. Dudarev, S. L., Botton, G. a., Savrasov, S. Y., Humphreys, C. J. \& Sutton, a. P. Electron-energyloss spectra and the structural stability of nickel oxide: An LSDA+U study. Phys. Rev. B 57, 1505-1509 (1998).

51. Maintz, S., Deringer, V. L., Tchougréeff, A. L. \& Dronskowski, R. Analytic projection from planewave and PAW wavefunctions and application to chemical-bonding analysis in solids. J. Comput. Chem. 34, 2557-67 (2013).

52. Deringer, V. L., Tchougr, A. L. \& Dronskowski, R. Crystal Orbital Hamilton Population ( COHP ) Analysis As Projected from Plane-Wave Basis Sets. 5461-5466 (2011). 
53. Dronskowski, R. \& Bloechl, P. E. Crystal orbital Hamilton populations (COHP): energy-resolved visualization of chemical bonding in solids based on density-functional calculations. J. Phys.

Chem. 97, 8617-8624 (1993).

Figure captions:

Figure 1 Redox behavior and $\mathrm{pH}$ as an energy ladder for triggering surface oxidation Cyclic voltammetry for a) $\mathrm{La}_{2} \mathrm{LiRuO}_{6}$ and b) $\mathrm{La}_{2} \mathrm{LilrO}_{6}$ at $\mathrm{pH}=13(0.1 \mathrm{M} \mathrm{KOH}), \mathrm{pH}=7\left(1 \mathrm{M} \mathrm{KNO}_{3}\right)$ and $\mathrm{pH}=0.3$ $\left(0.5 \mathrm{M} \mathrm{H}_{2} \mathrm{SO}_{4}\right.$ ) showing the activation process and the increase of the OER activity when reaching the bulk oxidation potential determined in organic solvent versus $\mathrm{Li}^{+} / \mathrm{Li}$. While the oxidation of $\mathrm{La}_{2} \mathrm{LiRuO}_{6}$ in acidic media leads to the irreversible loss of $\mathrm{RuO}_{4}^{-}$, the oxidation of $\mathrm{La}_{2} \mathrm{LillO}_{6}$ in similar conditions leads to a stable and large OER activity. For each curve, the first cycle is reported. c) Schematic energy level diagram showing the potential vs. $\mathrm{Li}^{+} / \mathrm{Li}$ found for $\mathrm{r}^{5+}$ oxidation in $\mathrm{La}_{2} \mathrm{LilrO}_{6}$ aligned with the water oxidation potential at different $\mathrm{pH}$ and compared with the oxidation potential of $\mathrm{Ir}^{4+}$ found for $\operatorname{IrO}_{2}\left(\operatorname{Ref}^{24}\right)$ or $\mathrm{Li}_{2} \mathrm{IrO}_{3}\left(\operatorname{Ref}^{42}\right)$ (see Supplementary Discussion for details about the potentials).

Figure 2 Oxygen evolution activity and surface redox active sites. a) Tafel plots for weight normalized $\mathrm{La}_{2} \mathrm{LillO}_{6}$ compared with nanoparticles of $\mathrm{IrO}_{2}$ at $\mathrm{pH}=1$ and $\mathrm{pH}=13$ (both using a loading of $\left.0.25 \mathrm{mg} / \mathrm{cm}^{2}\right)$ and b) Tafel plots for surface normalized $\mathrm{La}_{2} \mathrm{LilrO}_{6}\left(1.7 \mathrm{~m}^{2} / \mathrm{g}\right)$ compared with $\mathrm{IrO}_{2}$ nanoparticles $(7 \mathrm{~nm})$ extracted from reference $23^{23}$ and commercial micron-size $\mathrm{IrO}_{2}\left(1.8 \mathrm{~m}^{2} / \mathrm{g}\right)$ (error bars represent the standard deviation obtained from at least 3 independent measurements) In inset is shown a scanning electron miscroscopy image of pristine $\mathrm{La}_{2} \mathrm{LilrO}_{6}$, scale bar corresponds to $3 \mu \mathrm{m}$. c) $1^{\text {st }}, 25^{\text {th }}$ and $50^{\text {th }}$ cyclic voltammograms recorded for $\mathrm{La}_{2} \mathrm{LillO}_{6}$ showing the redox associated with $\mathrm{La}_{2} \mathrm{LilrO}_{6}$ delithiation occurring at $1.45 \mathrm{~V}$ vs. $\mathrm{RHE}$ and preceding the OER potential (> $1.5 \mathrm{~V}$ vs. RHE). Upon cycling, a pseudo-capacitive behavior gradually appears at low potential $(0.5-1.4 \mathrm{~V}$ vs. $\mathrm{RHE}$ ), corresponding to hydrated $\mathrm{IrO}_{2}$ pseudocapacitive behavior. d) the evolution of the cathodic pseudocapacitive charge during cycling (1.1 to $1.5 \mathrm{~V}$ vs $\mathrm{RHE}$ ) in Ar-saturated $0.1 \mathrm{M} \mathrm{H}_{2} \mathrm{SO}_{4}$ as well as OER activity at $20 \mathrm{~A} / \mathrm{g}$ measured for the same electrode.

Figure 3 Formation of $\mathrm{IrO}_{2}$ nanoparticles upon cycling and gradual deformation of the bulk phase. Fast Fourier transformed (FFT) filtered HRTEM images along the [201] zone axis of the space group $\mathrm{P}_{2} / \mathrm{c}_{\mathrm{c}}$ of $\mathrm{La}_{2} \mathrm{LilrO}_{6}: \mathbf{a}$ ) and $\left.\mathbf{b}\right)$ pristine, c) and d) after 1 cycle, e) and f) after 25 cycles and $\mathbf{g}$ ) and $\mathbf{h}$ ) after 50 cycles. The insets at the top left in panels a, c, d, e and f show the FFT patterns for each corresponding HRTEM image and can be indexed to the $\mathrm{P} 2_{1} / \mathrm{C}$ space group. The insets at the bottom left in panels $a, c, d$, e and $f$ show zoomed-in images of the same region (insets are $3.1 \mathrm{~nm}$ by side). 
The pristine and 1 cycle samples show crystalline $\mathrm{P} 2_{1} / \mathrm{c}$ symmetry, the FFT pattern of 25 cycles shows the same basic reflections as the pristine FTT. The filtered HRTEM image after 25 cycles shows a random distribution of local bright and dark zones (or channels, highlighted by white arrows). In addition to the basic reflections, the FFT pattern after 50 cycles shows super-reflections (white arrows) corresponding to the arrangement of rows of atoms with darker brightness shown in the HRTEM image (white arrows). In f) and h) two selected examples of TEM images after 25 and 50 cycles, respectively, are shown in the insets to illustrate the distribution of nanoparticles on the surface of each compound. The inter-atomic layer distances are in agreement with those of $\mathrm{IrO}_{2}$ (red squares).

Figure 4 Electronic structure and anionic redox involved into the oxidation process. (a) Computed density of states (DOS), (b) the crystal orbital overlap populations (COOPs) and (c) positive Fukui function for $\mathrm{La}_{2} \mathrm{LillO}_{6}$, rendering the computed electron density maps. The cyan volume corresponds to electron density depletion (holes) while the yellow regions correspond to electron density accumulation (electrons) due to orbital polarization. (d) DOS, (e) COOPs and (f) positive Fukui function for $\mathrm{La}_{2} \mathrm{IO}_{6}$. The calculations demonstrate the involvement of $\mathrm{t}_{2 \mathrm{~g}}$-like $\pi^{*} \mathrm{Ir}-\mathrm{O}$ antibonding states in the oxidation process (delithiation) of $\mathrm{La}_{2} \mathrm{LilrO}_{6}$ to form $\mathrm{La}_{2} \mathrm{IO}_{6}$. Nonbonding oxygen states are also found to participate for $\mathrm{La}_{2} \mid \mathrm{IO}_{6}$ as highlighted by the red circles in the Fukui function. The COOPs are computed for the Ir-O bonds (between 1.8 and $2.2 \AA$ ) and for the O-O bond (between 2.2 and $2.4 \AA$ ). The Fukui function confirms the polarization of the $\mathrm{t}_{2 \mathrm{~g}}$-like $\pi^{*}$ orbitals for $\mathrm{La}_{2} \mathrm{LilO}_{6}$ and $\mathrm{La}_{2} \mid \mathrm{IO}_{6}$ but also of non-bonding oxygen states for $\mathrm{La}_{2} \mid \mathrm{IO}_{6} . \mathbf{g}$ ) Difference (substraction) of Bader charge for each oxygen atoms from the average Bader charge of bulk oxygen atoms as a function of the distance from the surface for the (100) surface (blue) and (101) surface (green) for $\mathrm{La}_{2} \mid \mathrm{IO}_{6}$.

Figure 5 Cationic and anionic migration upon oxidation causing dislocations in oxidized $\mathrm{La}_{2} \mathrm{LillO}_{6}$. HRTEM image after filtering the noise of a) pristine $\mathrm{La}_{2} \mathrm{LilrO}_{6}$ and b) $\mathrm{La}_{2} \mathrm{LilrO}_{6}$ after 50 cycles showing the local transformation on the atomic scale due to oxidation: the bright dots in the HRTEM of pristine projected along [201] correspond to cations (Ir, La and Li). After cycling, some of these dots become less bright as confirmed by the intensity profiles beneath the HRTEM images. The atomic density along two atomic rows is shown in $b$, with the green line corresponding to the green rectangle in the TEM image above and red line to the red rectangle. The inset in b) shows a calculated HRTEM image exhibiting similar intensity features to the experimental image. The calculation has been done by varying every two consecutive atomic rows the Ir occupancy with first $0 \%$, then $50 \%$, the $100 \%$ occupancy, eventually forming a superstructure shown with the white rectangle. In addition to the difference in contrast of dots corresponding to projected atomic column, darker domains are found in the colored HRTEM image in c) and highlighted by the white hexagons. d) Experimental HRTEM image corresponding to the inset in b). e) Corresponding simulated HRTEM image showing the electron density (from red being the highest electronic density to black being the lowest) and f) projection of the atomic structures corresponding to d). The crystallographic model is described in Supplementary Figure 10. g) HRTEM image of $\mathrm{La}_{2} \mathrm{LillO}_{6}$ after 50 cycles with the corresponding fast Fourier transform shown in inset. The atomic migration and $\mathrm{IrO}_{2}$ nanoparticle 
formation cause a high concentration of defects in the $\mathrm{La}_{2} \mathrm{LilO}_{2}$ phase. Edge dislocations are shown in by $\mathrm{T}$ signs with dislocations represented by circles.

Figure 6 Oxygen evolution mechanism on the surface of $\mathrm{La}_{2} \mathrm{LilrO}_{6}$ with oxidized surface oxygen as reactive site and bulk oxygen migration. a) Normalized Ir $\mathrm{L}_{111}$-edge XAS spectra for pristine $\mathrm{La}_{2} \mathrm{LillO}_{6}$ (blue) and samples held at $1.65 \mathrm{~V}$ vs RHE for 10 minutes in $0.1 \mathrm{M} \mathrm{H}_{2} \mathrm{SO}_{4}$ (green) and compared with $\mathrm{IrO}_{2}$ reference (red) and b) second derivative of the XAS spectra showing the shift in the XANES features due to redox processes occurring during the course of the oxidation. c) Tafel plot for $\mathrm{La}_{2} \mathrm{LilrO}_{6}$ at different $\mathrm{pH}$ (in $\mathrm{H}_{2} \mathrm{SO}_{4}$ solution) and d) $\mathrm{pH}$ dependence on the OER potential at different current densities. From these measurements, a reaction order for $\mathrm{H}^{+}$of 0.87 on the SHE scale and 0.31 on the RHE scale is obtained. e) Scheme representing the proposed OER mechanism in acid with (1) the activation step consisting of surface oxidation/delithiation, then the formation of O-O bond through (2) the reaction of surface oxygen with water followed by the deprotonation or (3) the direct pairing of two surface oxygen, both being followed by the oxygen release and the formation of oxygen vacancies that can be filled by (4) the bulk migration of lattice oxygen or (5) by water. 
Figure 1
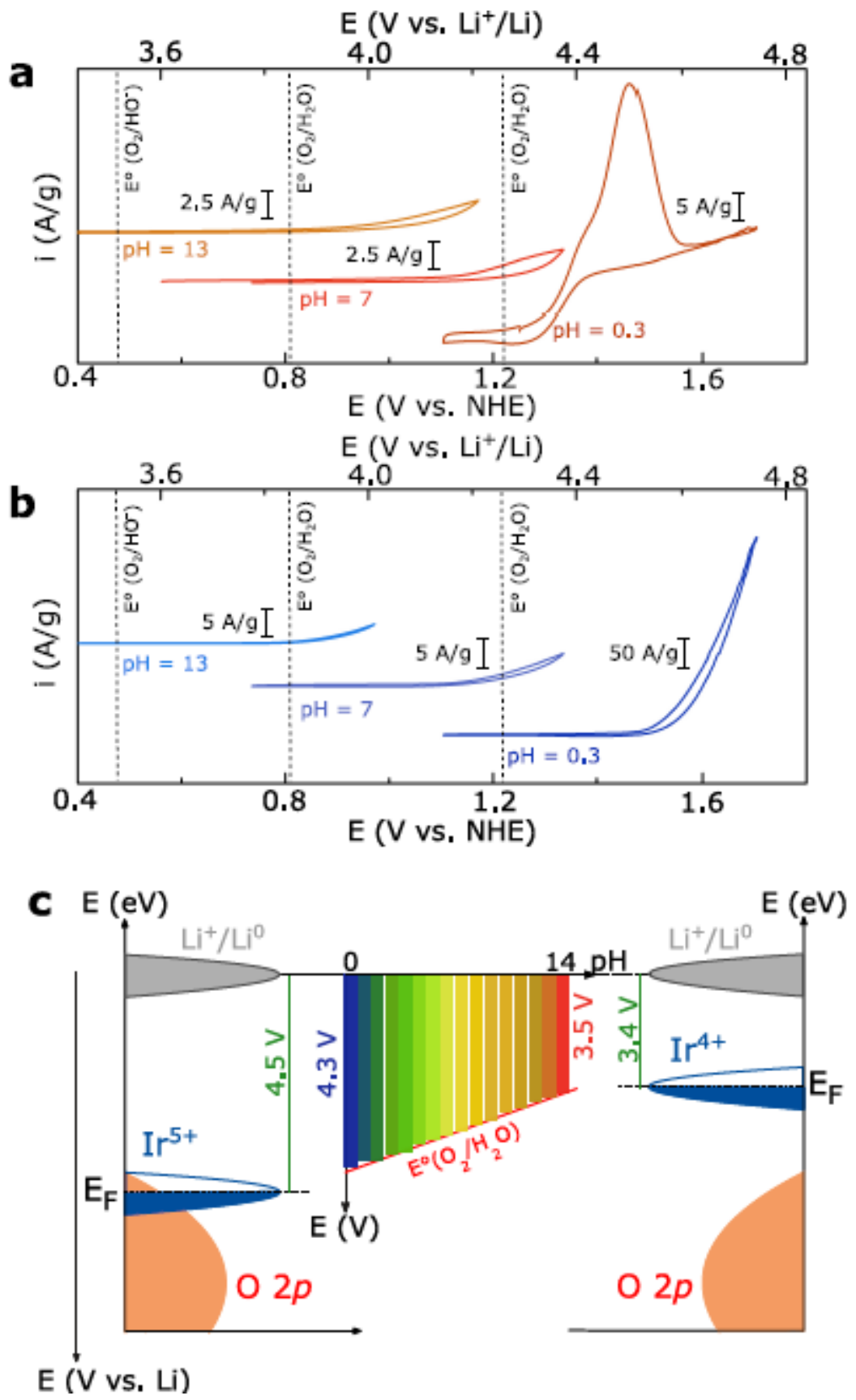
Figure 2
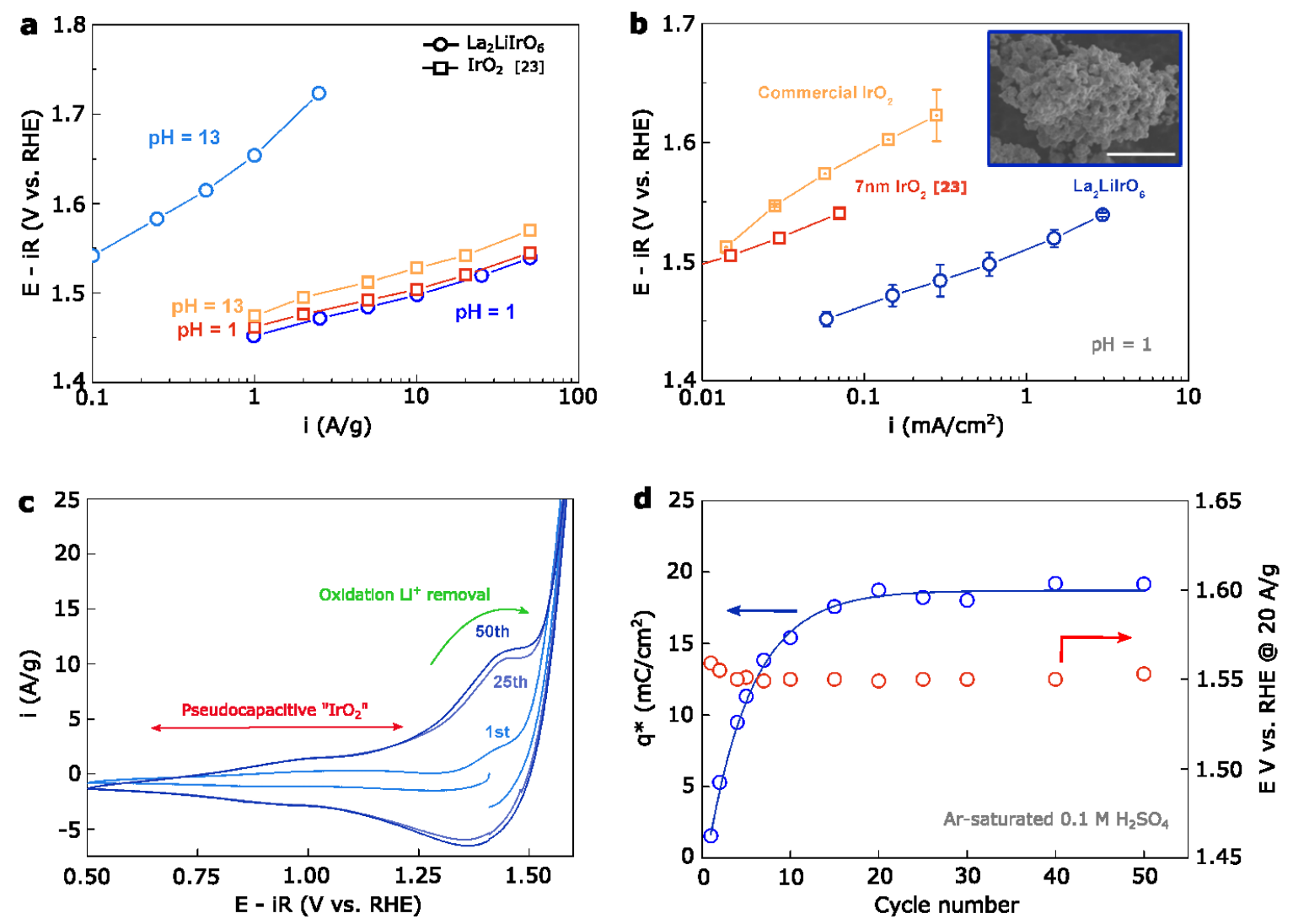
Figure 3

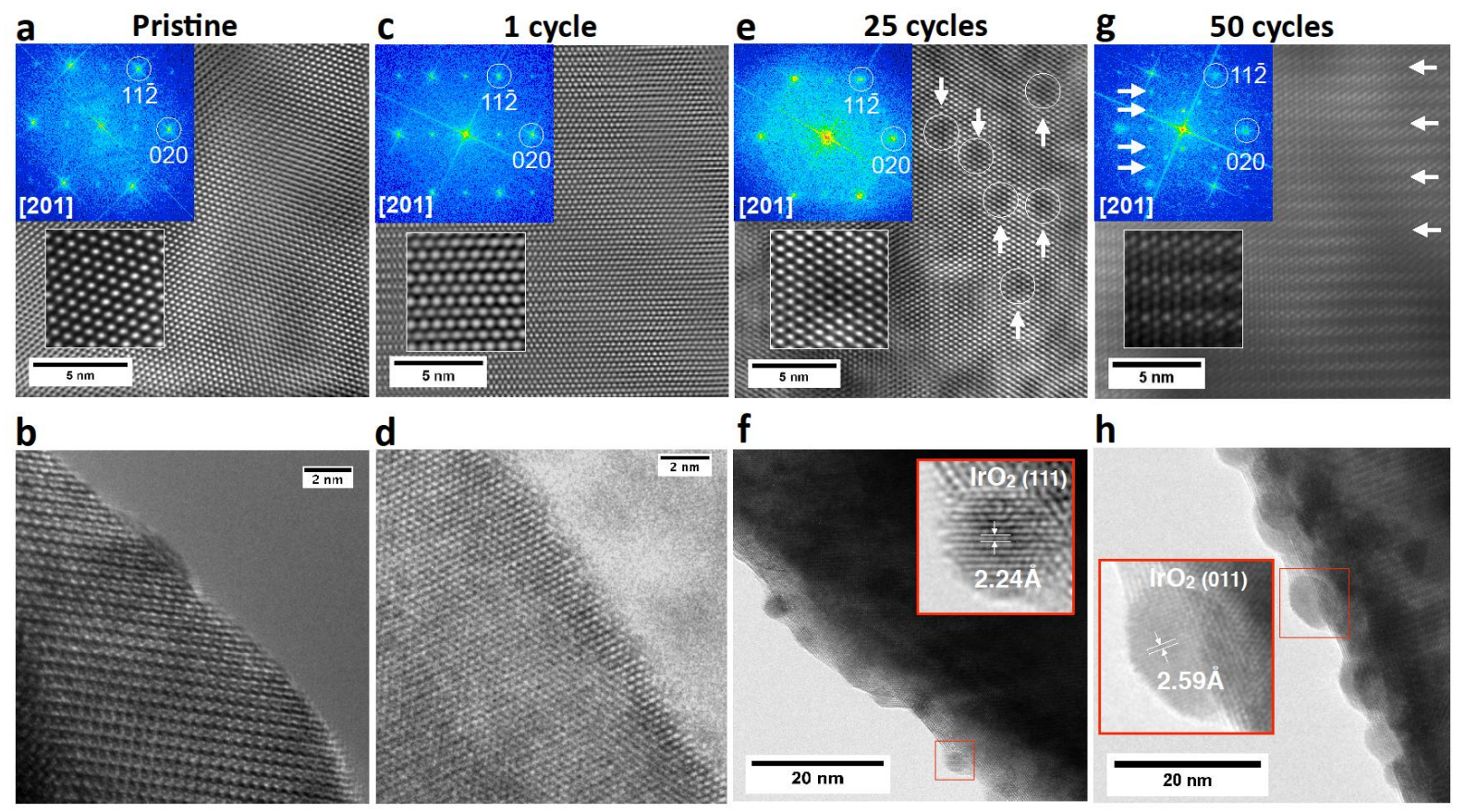


Figure 4

a

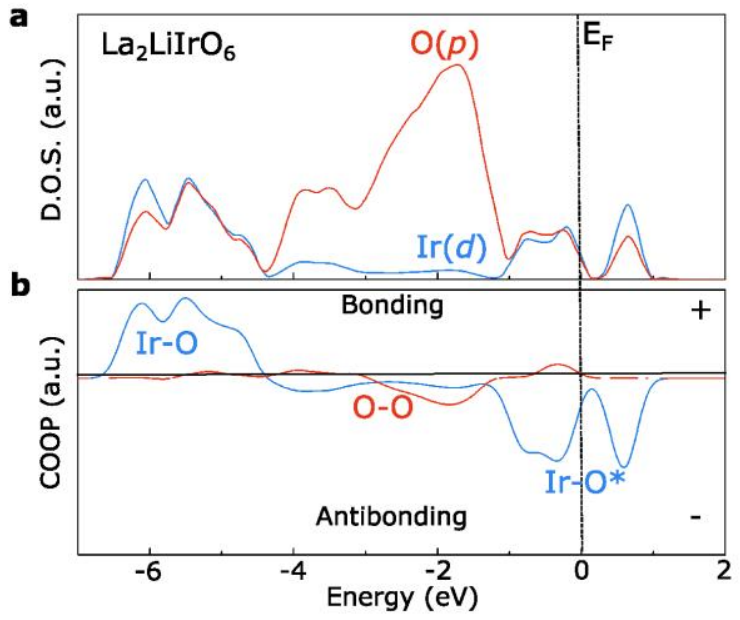

d

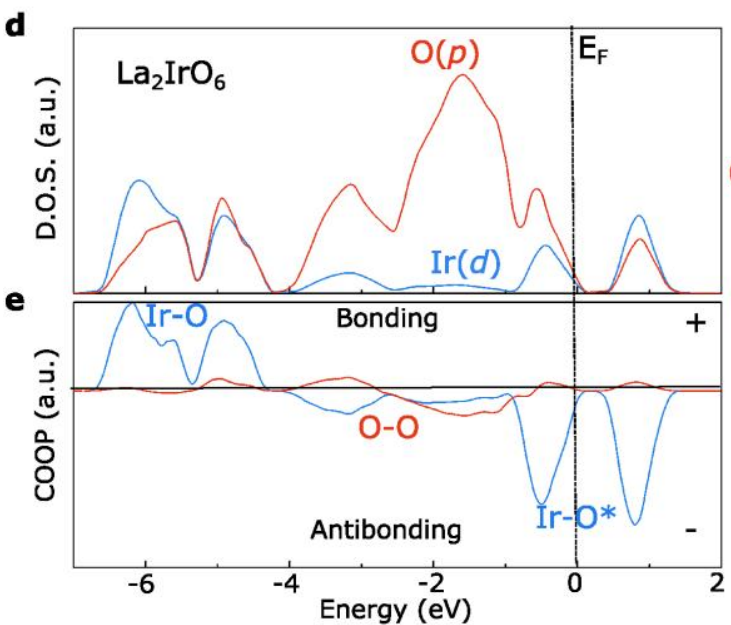

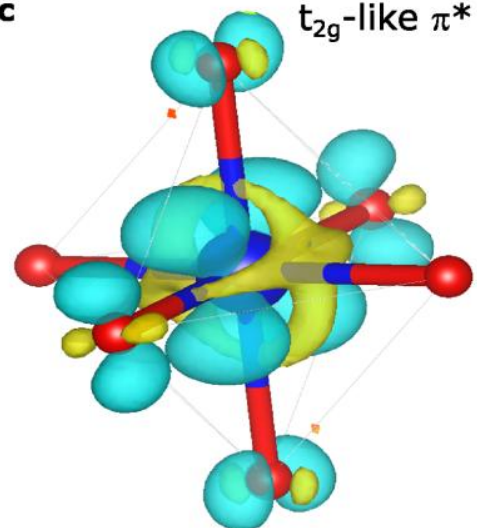

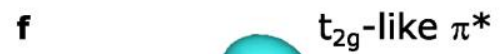

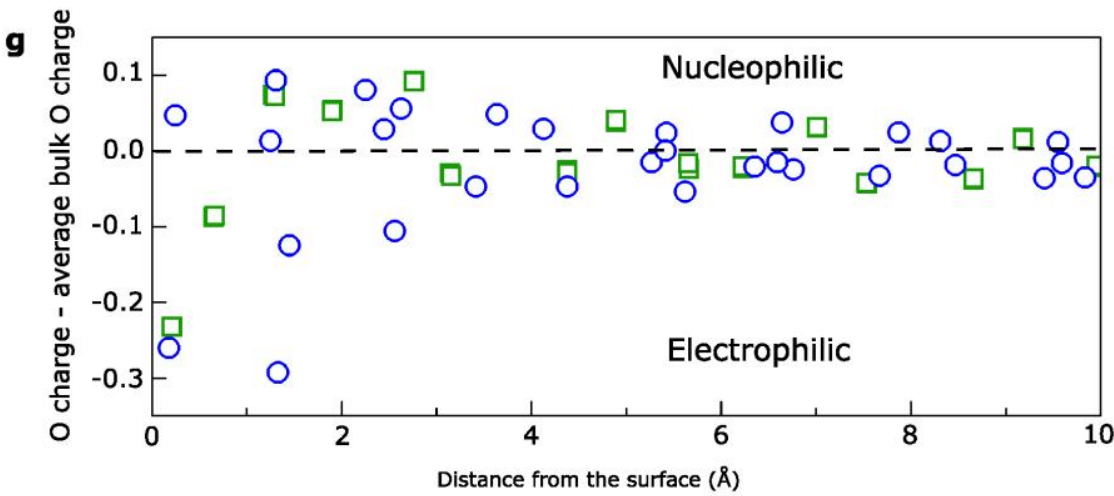


Figure 5
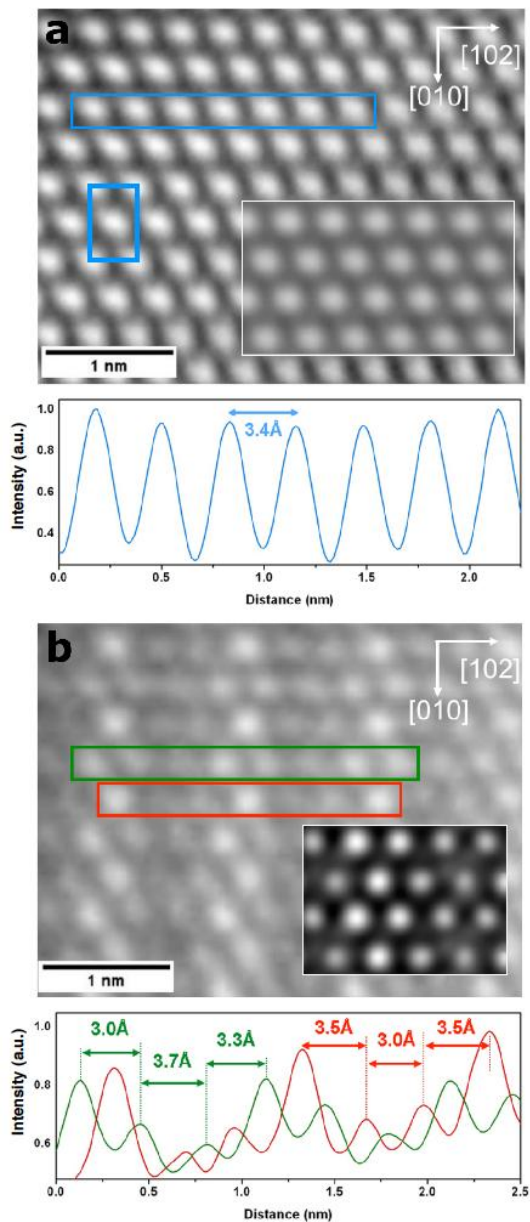
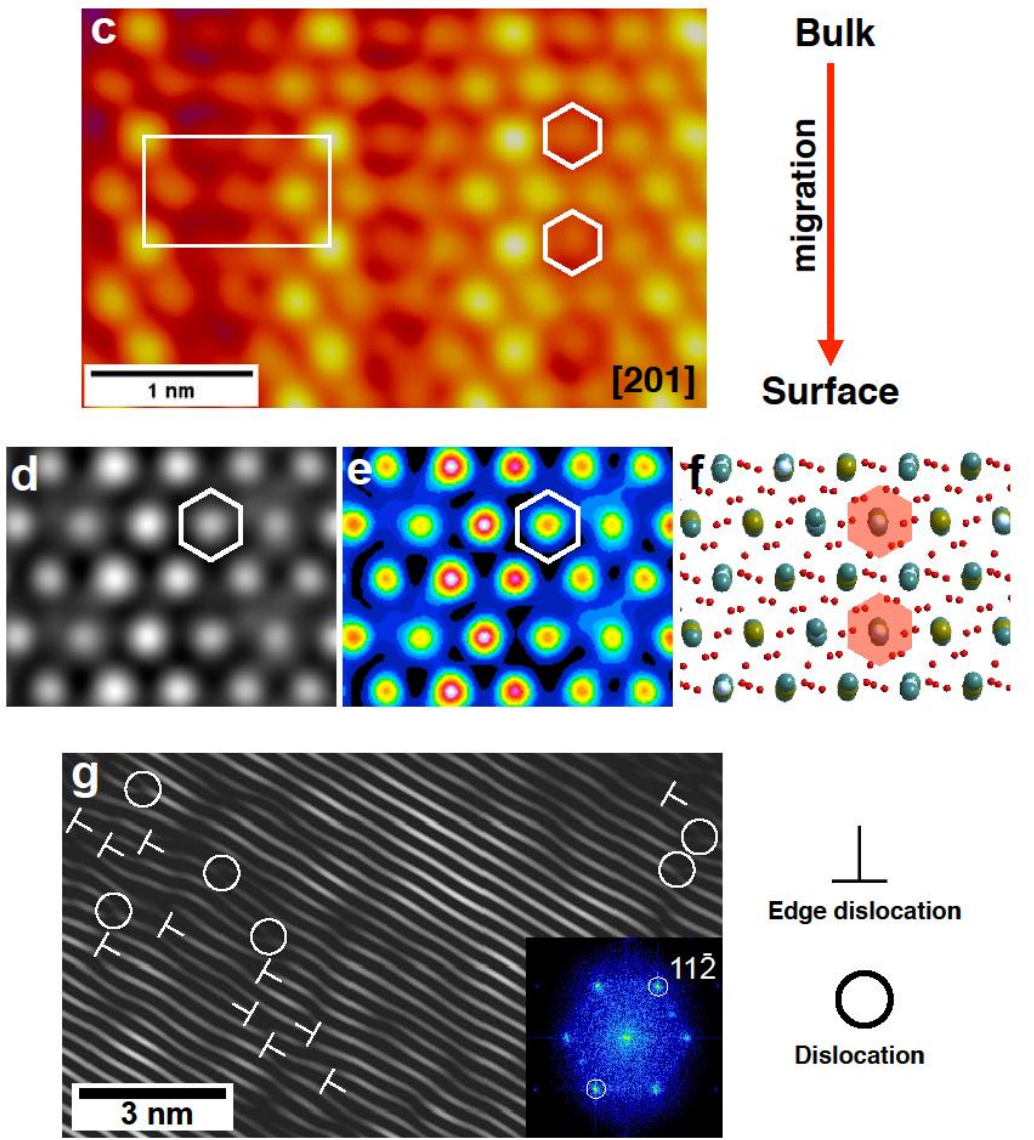

$\frac{1}{\text { Edge dislocation }}$

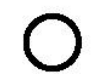

Dislocation 
Figure 6
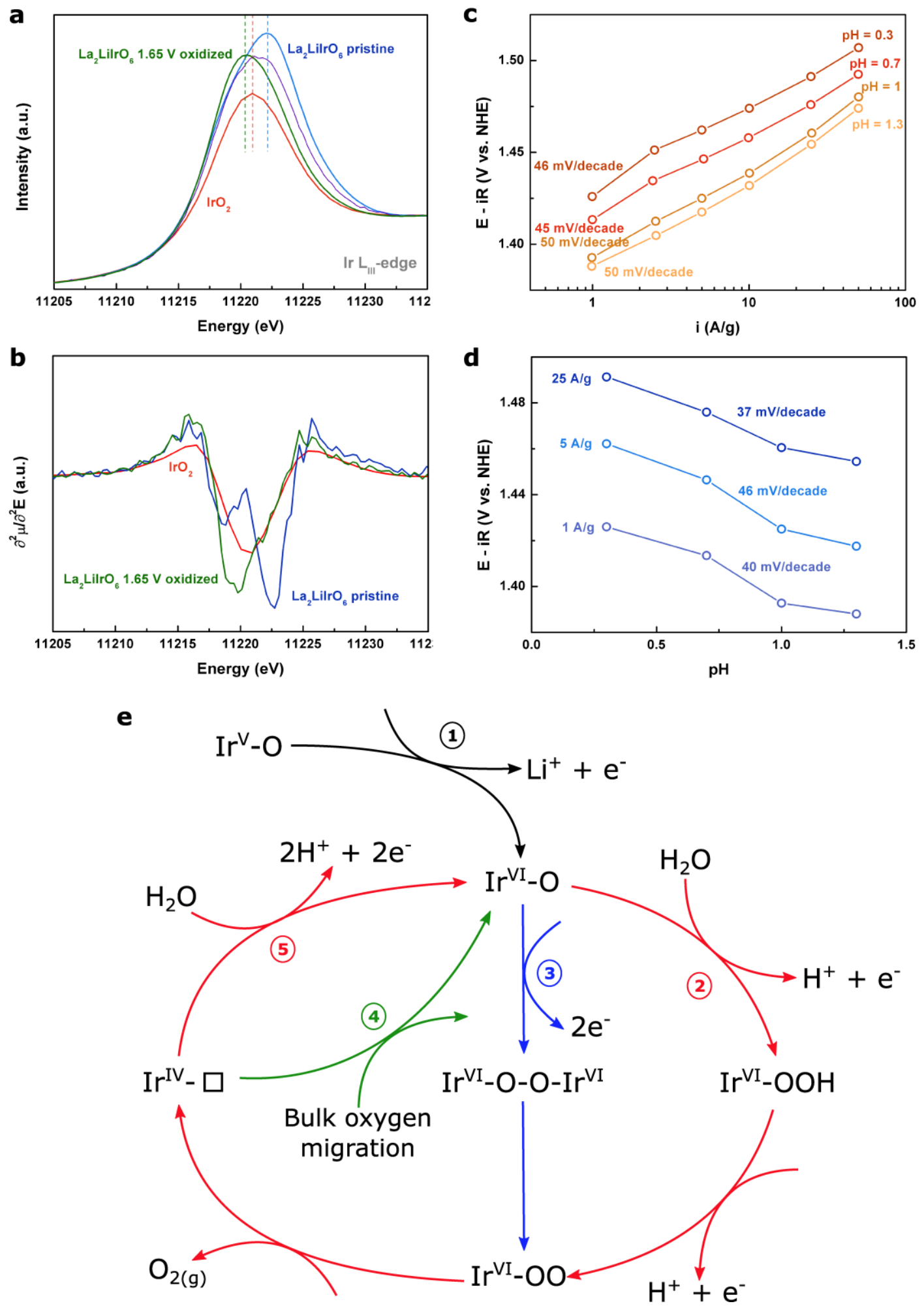